Journal for ImmunoTherapy of Cancer

To cite: Özdemir BC. Immune checkpoint inhibitor-related hypogonadism and infertility: a neglected issue in immunooncology. Journal for ImmunoTherapy of Cancer 2021;9:e002220. doi:10.1136/ jitc-2020-002220

Accepted 31 January 2021

Check for updates

(c) Author(s) (or their employer(s)) 2021. Re-use permitted under CC BY-NC. No commercial re-use. See rights and permissions. Published by BMJ.

${ }^{1}$ Department of Medical Oncology, Inselspital, Bern University Hospital, University of Bern, Bern, Switzerland

${ }^{2}$ International Cancer Prevention Institute, Lausanne, Switzerland

Correspondence to

Dr Berna C Özdemir;

berna.oezdemir@insel.ch

\title{
Immune checkpoint inhibitor-related hypogonadism and infertility: a neglected issue in immuno-oncology
}

\author{
Berna C Özdemir ${ }^{1,2}$
}

\begin{abstract}
Despite a significant amount of data on incidence and therapy of immune-related adverse events affecting virtually all organ systems, the potential impact of immune checkpoint inhibitors (ICls) on gonadal function has not been sufficiently studied. The limited evidence available suggests that ICl-related primary hypogonadism due to orchitis as well as secondary hypogonadism due to hypophysitis are a potential risk for infertility. A systematic investigation of gonadal function under ICls is warranted given the increasing application of ICls in the adjuvant setting, among young adults and children and the possible influence of sex hormone levels on the efficacy and toxicity of ICls.
\end{abstract}

Since the approval of ipilimumab in 2011, immune checkpoint inhibitors (ICIs) have significantly improved the treatment landscape of multiple cancer types. According to a recent analysis, approximately $43 \%$ of patients with cancer in the USA are eligible for ICIs, of whom $12 \%$ are estimated to respond. ${ }^{1}$ Nearly all patients undergoing ICIs will experience at least one type of immune-related adverse event (irAE), which can occur at any moment even months or years after therapy discontinuation. Moreover, these irAEs can affect any organ and be severe depending on the therapy regimen and underlying health condition of the patient, with $10 \%-30 \%$ of cases categorized as grades $3-5 .^{2}$ Endocrine toxicities are among the most common irAEs and, in contrast to other irAEs, tend to be irreversible and require life-long hormonal substitution. The most frequent endocrine complications are thyroid dysfunction $(30 \%)$, hypophysitis $(5.6 \%-11 \%)$, type I diabetes $(0.2 \%-2 \%)$, and adrenal insufficiency $(0.7 \%)$, although rare cases of hypoparathyroidism have also been described. ${ }^{3}$ Yet, surprisingly little is known about the potential impact of ICIs on gonadal function. A recent analysis of VigiBase, the WHO global database of individual case safety reports between 2011 and 2019, found a significant, disproportionately increased risk of hypogonadism for ICIs. Of the 13 reported cases of hypogonadism, 5 were classified as secondary and 1 as primary hypogonadism. ${ }^{4}$ A similar analysis of the French Pharmacovigilance database identified 94 cases of ICI-related hypophysitis, of whom $8 \%$ showed panhypopituitarism. ${ }^{5}$ Although panhypopituitarism can cause secondary hypogonadism, the levels of the pituitary gonadotropins follicle-stimulating hormone (FSH) and luteinizing hormone (LH) were rarely assessed and supplementation with gonadotropic hormones was mostly lacking. ${ }^{5}$

In a retrospective single-center review of 154 patients treated with ipilimumab, hypophysitis was found in $17(11 \%)$ of the patients and the anterior portion of the pituitary gland, responsible for gonadotropin secretion, was affected in all cases. The levels of $\mathrm{LH}, \mathrm{FSH}$ and testosterone were measured in 11 patients and were low, estradiol was not measured in the two female patients who were postmenopausal. The gonadal function normalized in two patients. ${ }^{6}$ Another center also reported an $11 \%$ incidence of hypophysitis under ipilimumab. The majority of the 15 affected patients presented gonadotropin deficiency $(80 \%, \mathrm{n}=12)$ that persisted in $16 \%$ of the cases at almost 3 years of follow-up. ${ }^{7}$

Even in the absence of hypophysitis, low total testosterone levels were reported in a retrospective single-center review of patients with melanoma. However, the actual incidence and extent of ICI-related hypogonadism remains unknown because measurements were not repeated nor performed systematically in all analyzed patients.

In addition to this central hypogonadism, ICI-induced inflammation of the gonads can be associated with impaired gonadal function, possibly leading to hypogonadism and infertility. Two cases have been reported of patients with metastatic melanoma who developed acute painful swelling of the testicles which turned out to be bilateral orchitis $^{9}$ and epididymo-orchitis ${ }^{10}$ under 
ipilimumab-nivolumab and pembrolizumab, respectively. While hormone measurements in the first case showed transient primary hypogonadism with low testosterone and high LH levels which recovered spontaneously, no hormone levels were measured in the second case which resolved after treatment with high-dose steroids. An analysis of 13 metastatic melanoma patients with testicular autopsy tissue samples showed that six of the seven men (86\%) who had received ICIs had impaired spermatogenesis compared with age-matched patients who were treatment naïve $(n=6) .{ }^{11}$ No data on potential effects on female fertility are currently available.

This lack of data is astonishing given the vast number of clinical trials performed over the last decade. For instance, none of the pivotal trials leading to Food and Drug Administration (FDA approval of ICIs in the different indications has provided information regarding fertility, menopause status, sex hormone levels, or sexual health-related quality of life. However, the assessment of gonadal function in reproductive-age men and women undergoing ICIs cannot be deferred any longer. ICIs are increasingly applied in the curative setting as adjuvant therapy where the risk of ICI-related hypogonadism, premature menopause or infertility, and their long-term consequences need to be balanced against the reduction of absolute risk of disease recurrence and discussed with the patient as it might affect the acceptance of such prophylactic therapies. In addition, the cancer incidence in young adults is increasing, with currently about $5 \%$ of all cancers being diagnosed in patients 20-39 years of age. It is therefore likely that in the future more reproductive-age men and women will be exposed to ICIs and need to be informed about the potential for gonadal toxicity. ${ }^{12}$ Similarly, multiple clinical trials are testing ICIs in pediatric patients and ICIs are already approved for several indications in this population. The current state of evidence does not provide any meaningful conclusions that can be used to counsel patients and their families regarding the risk of hypogonadism and its potential effects on the children's physical and mental development.

Even in the metastatic setting, patients need to be informed about potential hypogonadism or infertility considering that a subpopulation of patients will achieve durable, complete remission and might consider having children. More recently, ICIs have been incorporated into the standard of care in women with advanced breast cancer. Indefinite ovarian function suppression is a cornerstone of the management of premenopausal women with endocrine responsive advanced breast cancer. As such, it is important to determine whether ICIs affect ovarian function and future fertility in order to provide appropriate guidance regarding fertility preservation and to adapt treatment schemes. Additionally, whether the occurrence of ICI-related hypogonadism has a prognostic and predictive value also needs to be determined.

Another important issue to consider is the teratogenic potential of ICIs. The PD-1/PD-L1 and the CTLA4/CD80/ CD86 pathway are essential to induce maternal tolerance and prevent rejection of the semi-allogenic fetus. In pregnant mice, treatment with anti-PD-L1 antibodies dramatically increases the rate of abortion (86\%) compared with spontaneous abortion ( $18 \%)$, by depleting regulatory $\mathrm{T}$ cells (Tregs).$^{13}$ Likewise, in pregnant cynomolgus monkeys which received nivolumab from the onset of organogenesis through delivery, increased abortion and premature neonatal death were observed. ${ }^{13}$ Therefore, the FDA advises women of childbearing age to use effective contraception during treatment with anti-CTLA4 and anti-PD-1 anti-PD-L1 antibodies for at least 3-5 months, respectively, after the last dose. ${ }^{14}$

Currently, we have only limited knowledge on the effect of checkpoint inhibitors on the outcome of pregnant patients with cancer and their offspring. Three cases of ICI therapy in pregnant women with metastatic melanoma, ${ }^{15-17}$ as well as two cases ${ }^{18} 19$ of successful pregnancy that was conceived during treatment with ICIs with anti-PD1 alone or in combination with anti-CTLA4, were reported. None of the infants showed any developmental anomalies, except for congenital hypothyroidism detected in one newborn, possibly representing a fetal irAE from maternal anti-PD1 exposure. ${ }^{18}$

Given that melanoma is the most common malignancy during pregnancy and approximately one-third of all women diagnosed with melanoma who will be treated with ICI are of childbearing age, ${ }^{20}$ investigation of the safety of ICIs in an international registry prospectively collecting data on pregnant patients with cancer treated with ICI could be of great clinical value. ${ }^{20}$

The perturbation of sex hormone levels at the pituitary or gonadal level is not solely an issue of fertility and sexual health but might also affect efficacy and toxicity of ICIs. It has been reported ${ }^{21}$ that men derive a greater benefit from ICIs compared with women, which could be attributable to many biological factors including differences in the host hormonal milieu that affect response to ICIs. ${ }^{22}$ A systematic investigation of the potential impact of ICIs on gonadal function could be done by retrospectively analyzing sex hormone levels in serum from patients included in randomized clinical trials on adjuvant ICIs. Additionally, there are numerous clinical trials testing neoadjuvant ICIs in locally advanced tumors without prior anticancer therapy, a potential gonadotoxic effect of ICIs could be prospectively determined in these patients. Also, pooled analysis of real world data from large centers applying ICIs could provide important information regarding the clinical relevance of ICI-related hypogonadism. For a proper estimation of its true incidence, systematic collection of a minimal dataset including menopause status and measurement of LH, FSH and estradiol or testosterone levels, respectively, for all patients before starting ICIs and at occurrence of an irAE and every 3 months during therapy as well as during follow-up should be done. In particular, patients presenting with hypophysitis need long-term monitoring for gonadotropin deficiency in order to map the risk of persistent hypogonadism. Considering that patients with 
cancer rarely report spontaneously on their sexual health, physicians should regularly inquire on signs of hypogonadism such as erectile dysfunction, decreased libido, menstrual irregularities, vaginal dryness and hot flushes in order to detect patients at risk.

Confirming or excluding a potential effect of ICIs on gonadal function will not only help improve the counseling and treatment of patients with cancer in their reproductive years and the quality of life of cancer survivors but also contribute to a better understanding of the influence of sex hormones on efficacy and toxicity of these therapies.

Acknowledgements I would like to thank Professor Gian-Paolo Dotto and Ms Cristina Espinosa da Silva for their critical reviews and useful comments.

Contributors I am the sole author of this manuscript.

Funding The authors have not declared a specific grant for this research from any funding agency in the public, commercial or not-for-profit sectors.

Competing interests None declared.

Patient consent for publication Not required

Provenance and peer review Not commissioned; externally peer reviewed.

Open access This is an open access article distributed in accordance with the Creative Commons Attribution Non Commercial (CC BY-NC 4.0) license, which permits others to distribute, remix, adapt, build upon this work non-commercially, and license their derivative works on different terms, provided the original work is properly cited, appropriate credit is given, any changes made indicated, and the use is non-commercial. See http://creativecommons.org/licenses/by-nc/4.0/.

\section{REFERENCES}

1 Haslam A, Prasad V. Estimation of the percentage of US patients with cancer who are eligible for and respond to checkpoint inhibitor immunotherapy drugs. JAMA Netw Open 2019;2:e192535.

2 Martins F, Sofiya L, Sykiotis GP, et al. Adverse effects of immunecheckpoint inhibitors: epidemiology, management and surveillance. Nat Rev Clin Oncol 2019;16:563-80.

3 Nogueira E, Newsom-Davis T, Morganstein DL. Immunotherapyinduced endocrinopathies: assessment, management and monitoring. Ther Adv Endocrinol Metab 2019;10:2042018819896182.

4 Bai X, Lin X, Zheng K, et al. Mapping endocrine toxicity spectrum of immune checkpoint inhibitors: a disproportionality analysis using the who adverse drug reaction database, VigiBase. Endocrine 2020;69:670-81.
5 Garon-Czmil J, Petitpain N, Rouby F, et al. Immune check point inhibitors-induced hypophysitis: a retrospective analysis of the French pharmacovigilance database. Sci Rep 2019;9:19419.

6 Faje AT, Sullivan R, Lawrence D, et al. Ipilimumab-induced hypophysitis: a detailed longitudinal analysis in a large cohort of patients with metastatic melanoma. J Clin Endocrinol Metab 2014:99:4078-85.

7 Albarel F, Gaudy C, Castinetti F, et al. Long-Term follow-up of ipilimumab-induced hypophysitis, a common adverse event of the anti-CTLA-4 antibody in melanoma. Eur J Endocrinol 2015;172:195-204.

8 Ryder M, Callahan M, Postow MA, et al. Endocrine-Related adverse events following ipilimumab in patients with advanced melanoma: a comprehensive retrospective review from a single institution. Endocr Relat Cancer 2014;21:371-81.

9 Brunet-Possenti F, Opsomer MA, Gomez L, et al. Immune checkpoint inhibitors-related orchitis. Ann Oncol 2017;28:906-7.

10 Quach HT, Robbins CJ, Balko JM, et al. Severe epididymo-orchitis and encephalitis complicating anti-PD-1 therapy. Oncologist 2019;24:872-6.

11 Scovell JM, Benz K, Samarska I, et al. Association of impaired spermatogenesis with the use of immune checkpoint inhibitors in patients with metastatic melanoma. JAMA Oncol 2020;6:1297-9.

12 Miller KD, Fidler-Benaoudia F, Keegan TH. Cancer statistics for adolescents and young adults, 2020. CA Cancer J Clin 2020.

13 Poulet FM, Wolf JJ, Herzyk DJ, et al. An evaluation of the impact of PD-1 pathway blockade on reproductive safety of therapeutic PD-1 inhibitors. Birth Defects Res B Dev Reprod Toxicol 2016;107:108-19.

14 Walter JR, Xu S, Paller AS, et al. Oncofertility considerations in adolescents and young adults given a diagnosis of melanoma: fertility risk of food and drug Administration-approved systemic therapies. J Am Acad Dermatol 2016;75:528-34.

15 Mehta A, Kim KB, Minor DR. Case report of a pregnancy during ipilimumab therapy. J Glob Oncol 2018;4:1-3.

16 Burotto M, Gormaz JG, Samtani S, et al. Viable pregnancy in a patient with metastatic melanoma treated with double checkpoin immunotherapy. Semin Oncol 2018;45:164-9.

17 Menzer C, Beedgen B, Rom J, et al. Immunotherapy with ipilimumab plus nivolumab in a stage IV melanoma patient during pregnancy. Eur J Cancer 2018;104:239-42.

$18 \mathrm{Xu} \mathrm{W}$, Moor RJ, Walpole ET, et al. Pregnancy with successful foetal and maternal outcome in a melanoma patient treated with nivolumab in the first trimester: case report and review of the literature. Melanoma Res 2019;29:333-7.

19 Bucheit AD, Hardy JT, Szender JB, et al. Conception and viable twin pregnancy in a patient with metastatic melanoma while treated with CTLA-4 and PD-1 checkpoint inhibition. Melanoma Res 2020;30:423-5.

20 Andersson TM-L, Johansson ALV, Fredriksson I, et al. Cancer during pregnancy and the postpartum period: a population-based study. Cancer 2015;121:2072-7.

21 Conforti F, Bagnardi V, Pas TD. Cancer immunotherapy efficacy and patients' sex: a systematic review and meta-analysis. Lancet Oncol 2018. doi:10.1016/S1470-2045(18)30261-4

22 Özdemir BC, Dotto G-P. Sex hormones and anticancer immunity. Clin Cancer Res 2019;25:4603-10. 